\title{
Folding and Fundamental Groups of Flat Robertson-Walker Space
}

\author{
A. E. El-Ahmady* \\ Mathematics Department, Faculty of Science, Taibah University, Madinah, Saudi Arabia \\ Mathematics Department, Faculty of Science, Tanta University, Tanta, Egypt; a_elahmady@hotmail.com
}

\begin{abstract}
This paper aims to give a combinatorial characterization and also construct representations of the fundamental groups of the submanifolds on flat Robertson-Walker space by using some geometrical transformations. The homotopy groups of the limit folding on flat Robertson-Walker space are presented. The homotopy groups of the retractions and deformation retract on flat Robertson-Walker space are obtained. The fundamental groups of some types of geodesics in the flat Robertson-Walker space are discussed. New types of homotopy maps are deduced. Theorems governing this connection are achieved.
\end{abstract}

Keywords: Flat Robertson-Walker space $W^{4}$, Homotopy Groups, Foldings, Retractions, Deformation Retracts.

2000 Mathematics Subject Classification: 53A35, 51H20, 57N10.

\section{Introduction and Definitions}

Robertson-Walker space represents one of the most intriguing and emblematic discoveries in the history of geometry. Although if it were introduced for a purely geometrical purpose, they came into prominence in many branches of mathematics and physics. This association with applied science and geometry generated synergistic effect: applied science gave relevance to Robertson-Walker space and Robertson-Walker space allowed formalizing practical problems [14-19, 20,21].

In vector spaces and linear maps; topological spaces and continuous maps; groups and homomorphisms together with the distinguished family of maps is referred to as a category. An operator which assigns to every object in one category a corresponding object in another category and to every map in the first a map in the second in such a way that compositions are preserved and the identity map is taken to the identity map is called a functor. Thus, we may summarize our activities thus far by saying that we have constructed a functor (the fundamental group functor) from the category of pointed spaces and maps to the category of groups and homomorphisms. Such functors are the vehicles by which one translates topological problems into algebraic problem $[8,10,12,22-25]$.

Most folding problems are attractive from a pure mathematical standpoint, for the beauty of the problems themselves. The folding problems have close connections to important industrial applications. Linkage folding has applications in robotics and hydraulic tube bending. Paper folding has application in sheet-metal bending, packaging, and air -bag folding [9]. Also, used folding to solve difficult problems related to shell structures in civil engineering and aero space design, namely buckling instability [11]. Isometric folding between two Riemannian manifold may be characterized as maps that send piecewise geodesic segments to a piecewise geodesic segments of the same length [4]. For a topological folding the maps do not preserves lengths $[5,6]$, i.e. A map $\mathfrak{I}: M \rightarrow N$, where $M$ and $N$ are $C^{\infty}$-Riemannian manifolds of dimension $m, n$ respectively is said to be an isometric folding of $\mathrm{M}$ into $\mathrm{N}$, iff for any piecewise geodesic path $\gamma: J \rightarrow M$, the induced path

* Corresponding author:

A. E. El-Ahmady (a_elahmady@hotmail.com) 
$\mathfrak{I}^{\circ} \gamma: J \rightarrow \mathrm{N}$ is a piecewise geodesic and of the same length as $\gamma$. If $\mathfrak{I}$ does not preserve length, then $\mathfrak{I}$ is a topological folding $[1,2,3,7]$.

A subset $A$ of a topological space $X$ is called a retract of $X$ if there exists a continuous map $r: X \rightarrow A$ such that $r(a)=$ $a, \forall \mathrm{a} \in A$ where $A$ is closed and $X$ is open $[13,18]$. Also, let $X$ be a space and $A$ a subspace. A map $r: X \rightarrow A$ such that $r(a)=a$, for all $a \in A$, is called a retraction of $X$ onto $A$ and $A$ is the called a retract of $X$. This can be re stated as follows. If $i: A \rightarrow X$ is the inclusion map, then $r: X \rightarrow A$ is a map such that $r i=i d_{A}$ If, in addition, $r i \simeq i d_{x}$, we call $r$ a deformation retract and $A$ a deformation retract of $X$. Another simple-but extremely useful-idea is that of a retract $[26,27,28]$. If $A, X \subset M$, then $A$ is a retract of $X$ if there is a commutative diagram.

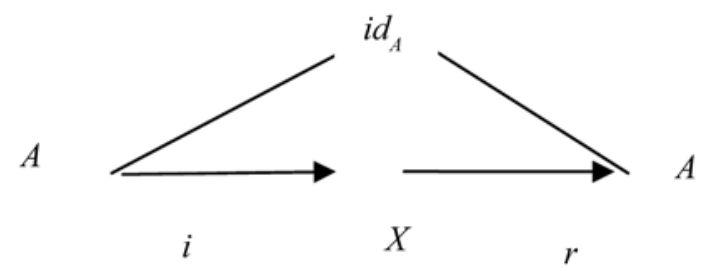

If $f: A \rightarrow B$ and $g: X \rightarrow Y$, then $f$ is a retract of $g$ if there is a commutative diagram $[10,29]$.

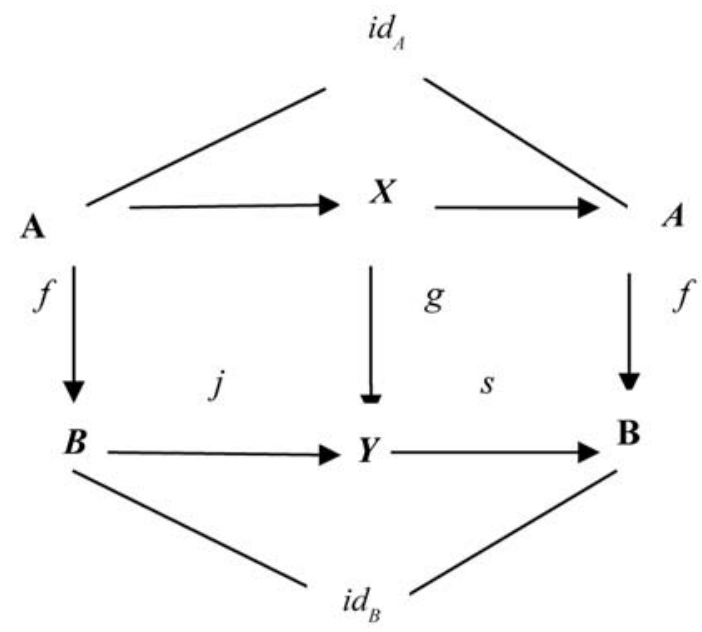

\subsection{Main Results}

THEOREM 1. The fundamental group of types of the retractions of flat Robertson-Walker space $\boldsymbol{w}^{4}$ are isomorphic to $\mathrm{Z}$ or to identity group.

Proof. Consider the flat Robertson -Walker space $w^{4}$ used spherical coordinate $t, r, \theta$ and $\varphi$ with metric

$$
d s^{2}=-d t^{2}+a^{2}(t)\left\{d r^{2}+r^{2}\left(d \theta^{2}+\sin ^{2} \theta d \varphi^{2}\right)\right\}
$$

The coordinates of flat Robertson-Walker space $w^{4}$ are given by

$\mathrm{X}_{1}=\mathrm{t}$

$\mathrm{X}_{2}=\mathrm{a}(\mathrm{t}) \mathrm{r} \sin \theta \cos \phi$

$\mathrm{X}_{3}=\mathrm{a}(\mathrm{t}) \mathrm{r} \sin \theta \sin \phi$

$X_{4}=a(t) r \cos \theta$

Now, we use Lagrangian equations $\frac{\mathrm{d}}{\mathrm{ds}}\left(\frac{\partial \mathrm{T}}{\partial \dot{\varphi}_{\mathrm{i}}}\right)-\frac{\partial \mathrm{T}}{\partial \varphi_{\mathrm{i}}}=0$ $i=1,2,3$. to find a geodesic which is a subset of the flat Robertson -Walker space $w^{4}$. Since

$$
\mathrm{T}=\frac{1}{2}\left\{-\mathrm{t}^{2}+\mathrm{a}^{2}(\mathrm{t})\left(\dot{\mathrm{r}}^{2}+\mathrm{r}^{2}\left(\dot{\theta}^{2}+\sin ^{2} \theta \dot{\phi}^{2}\right)\right)\right\}
$$

Then the Lagrangian equations for flat Robertson -Walker space $W^{4}$ are

$$
\begin{aligned}
& \frac{\mathrm{d}}{\mathrm{ds}}\left(\mathrm{t}^{\prime}\right)+\mathrm{a}(\mathrm{t})\left(\mathrm{r}^{2}+\mathrm{r}^{2}\left(\hat{\theta}^{2}+\sin ^{2} \theta \hat{\phi}^{2}\right)=0\right. \\
& \frac{\mathrm{d}}{\mathrm{ds}}\left(\mathrm{a}^{2}(\mathrm{t}) \mathrm{r}^{2} \dot{\theta}^{\prime}\right)-\mathrm{a}^{2}(\mathrm{t}) \mathrm{r}^{2} \sin \theta \cos \theta \phi^{\prime}=0 \\
& \frac{\mathrm{d}}{\mathrm{ds}}\left(\mathrm{a}^{2}(\mathrm{t}) \mathrm{r}^{2} \sin ^{2} \theta \dot{\phi}\right)=0
\end{aligned}
$$

From equation (5) we obtain $\left(\mathrm{a}^{2}(\mathrm{t}) \mathrm{r}^{2} \sin ^{2} \theta \phi\right)$ constant say $\beta_{1}$, if $\beta_{1}=0$, we obtain the following cases. If $\phi=0$, then the coordinate of flat Robertson -Walker space are given by $\left\{\mathrm{x}_{1}=\mathrm{t}, \mathrm{x}_{2}=\mathrm{a}(\mathrm{t}) \mathrm{r} \sin \theta, \mathrm{x}_{3}=0, \mathrm{x}_{4}=\mathrm{a}(\mathrm{t}) \mathrm{r} \cos \theta\right\}$, which is a hypersurface $\mathrm{F}_{1} \subset w^{4}$ in flat Robertson -Walker space which is a geodesic and a retraction. If $\phi=30$, then the coordinate of flat Robertson -Walker space are given by $\left\{\mathrm{x}_{1}=\mathrm{t}, \mathrm{x}_{2}=\right.$ $\left.\frac{\sqrt{3}}{2} \mathrm{a}(\mathrm{t}) \mathrm{r} \sin \theta, \mathrm{x}_{3}=\frac{1}{2} \mathrm{a}(\mathrm{t}) \mathrm{r} \sin \theta, \mathrm{x}_{4}=\mathrm{a}(\mathrm{t}) \mathrm{r} \cos \theta\right\}$ which is a hypersurface $\mathrm{F}_{2} \subset w^{4}$ in flat Robertson -Walker space which is a geodesic and a retraction. If $\phi=45$, then the coordinate of flat Robertson -Walker space are given by $\left\{\mathrm{x}_{1}=\mathrm{t}, \mathrm{x}_{2}=\frac{1}{\sqrt{2}} \mathrm{a}(\mathrm{t}) \mathrm{r} \sin \theta, \mathrm{x}_{3}=\frac{1}{\sqrt{2}} \mathrm{a}(\mathrm{t}) \mathrm{r} \sin \theta, \mathrm{x}_{4}=\right.$ $\mathrm{a}(\mathrm{t}) \mathrm{r} \cos \theta\}$ which is a hypersurface $\mathrm{F}_{3} \subset w^{4}$ in flat Robertson -Walker space which is a geodesic and a retraction. If $\phi=60$, then the coordinate of flat Robertson -Walker space are given by $\left\{\mathrm{X}_{1}=\mathrm{t}, \mathrm{x}_{2}=\frac{1}{2} \mathrm{a}(\mathrm{t}) \mathrm{r} \sin \theta, \mathrm{x}_{3}=\frac{\sqrt{3}}{2} \mathrm{a}(\mathrm{t})\right.$ $\left.\mathrm{r} \sin \theta, \mathrm{x}_{4}=\mathrm{a}(\mathrm{t}) \mathrm{r} \cos \theta\right\}$, which is a hypersurface $\mathrm{F}_{4} \subset w^{4}$ in flat Robertson -Walker space which is a geodesic and a retraction. 
If $\phi=90$, then the coordinate of flat Robertson -Walker space are given by $\left\{\mathrm{x}_{1}=\mathrm{t}, \mathrm{x}_{2}=0, \mathrm{x}_{3}=\mathrm{a}(\mathrm{t}) \mathrm{r} \sin \theta, \mathrm{x}_{4}=\mathrm{a}(\mathrm{t}) \mathrm{r} \cos \theta\right\}$, which is a hypersurface $\mathrm{F}_{5} \subset w^{4}$ in flat Robertson -Walker space which is a geodesic and a retraction. If $\phi=180$, then the coordinate of flat Robertson -Walker space are given by $\left\{\mathrm{x}_{1}=\mathrm{t}, \mathrm{x}_{2}=-\mathrm{a}(\mathrm{t}) \mathrm{r} \sin \theta, \mathrm{x}_{3}=0, \mathrm{x}_{4}=\mathrm{a}(\mathrm{t}) \mathrm{r} \cos \theta\right\}$, which is a hypersurface $\mathrm{F}_{6} \subset w^{4}$ in flat Robertson Walker space which is a geodesic and a retraction. If $\theta=0$, then the coordinate of flat Robertson -Walker space are given by $\left\{\mathrm{x}_{1}=\mathrm{t}, \mathrm{x}_{2}=0, \mathrm{x}_{3}=0, \mathrm{x}_{4}=\mathrm{a}(\mathrm{t}) \mathrm{r}\right\}$, which is a hypersurface $\mathrm{F}_{7} \subset w^{4}$ in flat Robertson -Walker space which is a geodesic and a retraction. If $\theta=30$, then the coordinate of flat Robertson -Walker space are given by $\left\{\mathrm{x}_{1}=\mathrm{t}, \mathrm{x}_{2}=\frac{1}{2} \mathrm{a}(\mathrm{t}) \mathrm{r} \cos \phi, \mathrm{x}_{3}=\frac{1}{2} \mathrm{a}(\mathrm{t}) \mathrm{r} \sin \phi, \mathrm{x}_{4}=\frac{\sqrt{3}}{2} \mathrm{a}(\mathrm{t}) \mathrm{r}\right\}$, which is a hypersurface $\mathrm{F}_{8} \subset w^{4}$ in flat Robertson -Walker space which is a geodesic and a retraction. If $\theta=45$, then the coordinate of flat Robertson -Walker space are given by $\left\{\mathrm{x}_{1}=\mathrm{t}, \mathrm{x}_{2}=\frac{1}{\sqrt{2}} \mathrm{a}(\mathrm{t}) \mathrm{r} \cos \phi, \mathrm{x}_{3}=\frac{1}{\sqrt{2}} \mathrm{a}(\mathrm{t}) \mathrm{r} \sin \phi\right.$, $\left.\mathrm{x}_{4}=\frac{1}{\sqrt{2}} \mathrm{a}(\mathrm{t}) \mathrm{r}\right\}$, which is a hypersurface $\mathrm{F}_{9} \subset w^{4}$ in flat Robertson-Walkerspace whichisa geodesic and a retraction. If $\theta=60$, then the coordinate of flat Robertson -Walker space are given by $\left\{\mathrm{x}_{1}=\mathrm{t}, \mathrm{x}_{2}=\frac{\sqrt{3}}{2} \mathrm{a}(\mathrm{t}) \mathrm{r} \cos \phi, \mathrm{x}_{3}=\frac{\sqrt{3}}{2} \mathrm{a}(\mathrm{t}) \mathrm{r} \sin \phi\right.$, $\left.\mathrm{x}_{4}=\frac{1}{2} \mathrm{a}(\mathrm{t}) \mathrm{r}\right\}$, which is a hypersurface $\mathrm{F}_{10} \subset w^{4}$ in flat Robertson -Walker space which is a geodesic and a retraction. If $r=0$, then the coordinate of flat Robertson -Walker space are given by $\left\{\mathrm{x}_{1}=\mathrm{t}, \mathrm{x}_{2}=0, \mathrm{x}_{3}=0, \mathrm{x}_{4}=0\right\}$, which is a point $P_{1} \subset w^{4}$ in flat Robertson -Walker space which is a minimal geodesic and a minimal retraction. If $\mathrm{t}=0$, then the coordinate of flat Robertson -Walker space are given by $\left\{\mathrm{x}_{1}=0, \mathrm{x}_{2}=\mathrm{a}(\mathrm{t}) \mathrm{r} \sin \theta \cos \phi, \mathrm{x}_{3}=\mathrm{a}(\mathrm{t}) \mathrm{r} \sin \theta \sin \phi, \mathrm{x}_{4}=\right.$ $\mathrm{a}(\mathrm{t}) \mathrm{r} \cos \theta\}$, which is a sphere $\mathrm{S}^{2} \subset w^{4}$ in flat Robertson Walker space which is a geodesic and a retraction. If $t=0$, and $\theta=0$, then the coordinate of flat Robertson -Walker space are given by $\left\{\mathrm{x}_{1}=0, \mathrm{x}_{2}=0, \mathrm{x}_{3}=0, \mathrm{x}_{4}=\mathrm{a}(\mathrm{t}) \mathrm{r}\right\}$, which is a point $P_{2} \subset w^{4}$ in flat Robertson -Walker space which is a minimal retraction and a minimal geodesic. If $\mathrm{t}=0$, and $\phi=0$, then the coordinate of flat Robertson Walker space are given by $\left\{\mathrm{x}_{1}=0, \mathrm{x}_{2}=\mathrm{a}(\mathrm{t}) \mathrm{r} \sin \theta, \mathrm{x}_{3}=0\right.$, $\left.\mathrm{x}_{4}=\mathrm{a}(\mathrm{t}) \mathrm{r} \cos \theta\right\}$ which is a circle $\mathrm{S}^{1} \subset w^{4}$ in flat Robertson Walker space which is a geodesic and a retraction. Thus, $\pi_{1}\left\{w^{4}-\left(\mu_{i}\right)\right\} \approx \pi_{1}\left(\mathrm{~F}_{\mathrm{i}}, i=1-10\right), \pi_{1}\left\{w^{4}-\left(\mu_{i}\right)\right\} \approx \pi_{1}\left(\mathrm{~S}^{2}\right)$, $\pi_{1}\left\{w^{4}-\left(\mu_{i}\right)\right\} \approx \pi_{1}\left(P_{i}, i=1,2\right)$ and $\pi_{1}\left\{w^{4}-\left(\mu_{i}\right)\right\} \approx \pi_{1}\left(\mathrm{~S}^{1}\right)$. Therefore $\pi_{1}\left(\mathrm{~F}_{\mathrm{i}}\right), \pi_{1}\left(\mathrm{~S}^{2}\right), \pi_{1}\left(P_{i}, i=1,2\right)$ and $\pi_{1}\left(\mathrm{~S}^{1}\right)$ are isomorphic to the identity group or $\mathrm{Z}$.

Theorem 2. The fundamental groups of the minimal retractions induce the fundamental groups of the null geodesics and the fundamental groups are isomorphic to identity group.

Proof. Consider the geodesic $P_{1} \subset w^{4}$, where $\left\{\mathrm{x}_{1}, \mathrm{x}_{2}\right.$, $\left.\mathrm{x}_{3}, \mathrm{x}_{4}\right\}=\{t, 0,0,0\}$, it is a minimal retraction and null geodesic in $\left\{w^{4}-\left(\mu_{i}\right)\right\}$ also $\pi_{1}\left\{w^{4}-\left(\mu_{i}\right)\right\} \approx \pi_{1}\left(P_{1}\right)$ is isomorphic to identity group. Also, $P_{2} \subset w^{4}$ is a minimal retraction and null geodesic and $\pi_{1}\left\{w^{4}-\left(\mu_{i}\right)\right\} \approx \pi_{1}\left(P_{2}\right)$ is isomorphic to identity group.

Propsition 1. The fundamental groups of types of the deformation retractions of flat Robertson-Walker space $\left\{w^{4}-\left(\mu_{i}\right)\right\}$ are isomorphic to $\mathrm{Z}$ or to identity group.

Theorem 3. Under the defined folding and any folding homeomorphic to this type of folding, the fundamental group of the deformation retracts of the folded flat Robertson -Walker space i.e. $\pi_{1}\left(\eta \mathfrak{I}\left\{w^{4}-\left(\mu_{i}\right)\right\}\right)$ into the folded geodesics is the same as the fundamental group of the deformation retract of the flat Robertson -Walker space into the geodesics.

Proof. Let the deformation retract of flat Robertson Walker space $\left\{w^{4}-\left(\mu_{i}\right)\right\}$ is defined by $\eta:\left\{w^{4}-\left(\mu_{i}\right)\right\} \times$ $\mathrm{I} \rightarrow\left\{w^{4}-\left(\mu_{i}\right)\right\}$, where $\left\{w^{4}-\left(\mu_{i}\right)\right\}$ is the open flat Robertson Walker space and I is the closed interval $[0,1]$. The retractions of the open flat Robertson -Walker space $\left\{w^{4}-\left(\mu_{i}\right)\right\}$ are given by $\mathrm{F}_{\mathrm{i}}, \mathrm{i}=1,2, \ldots, 10, \mathrm{~S}^{2}, P_{1}, P_{2}, S^{1}$.

The fundamental group of the deformation retract for the flat Robertson -Walker space $\left\{w^{4}-\left(\mu_{i}\right)\right\}$ onto a geodesic $\mathrm{F}_{5} \subset\left\{w^{4}-\left(\mu_{i}\right)\right\}$ is given by

$$
\begin{aligned}
& \pi_{1}(\eta(m, c))=\cos \frac{\pi c}{2}\{\{t, a(t) r \sin \theta \cos \phi, \\
& \left.a(t) r \sin \theta \sin \phi, a(t) r \cos \theta\}-\left(\mu_{i}\right)\right\}+\frac{\sin \pi c}{2} \\
& \{t, 0, a(t) r \sin \theta, a(t) r \cos \theta\}
\end{aligned}
$$

where $\pi_{1}(\eta(m, 0))=\{t, a(t) r \sin \theta \cos \phi, a(t) r \sin \theta \sin \phi, a(t)$ $r \cos \theta\}$ is isomorphic to identity group, and $\pi_{1}(\eta(m, 1))=$ $\{\mathrm{t}, 0, \mathrm{a}(\mathrm{t}) \mathrm{r} \sin \theta, \mathrm{a}(\mathrm{t}) \mathrm{r} \cos \theta\}$. Thus, $\pi_{1}\left\{w^{4}-\left(\mu_{i}\right)\right\} \approx \pi_{1}\left(\mathrm{~F}_{5} \subset\right.$ $\left.\left\{w^{4}-\left(\mu_{i}\right)\right\}\right)$, is isomorphic to identity group. 
The fundamental group of the deformation retract for the flat Robertson -Walker space $\left\{w^{4}-\left(\mu_{i}\right)\right\}$ onto a geodesic $\mathrm{F}_{7} \subset\left\{w^{4}-\left(\mu_{i}\right)\right\}$ is given by

$$
\pi_{1}(\eta(m, c))=\frac{1-c}{1+c}\{\{t, a(t) r \sin \theta \cos \phi, a(t) r \sin \theta \sin
$$

), $\left.\mathrm{a}(\mathrm{t}) \mathrm{r} \cos \theta\}-\left(\mu_{i}\right)\right\}+\mathrm{c}(2 \mathrm{c}-1)\left\{\mathrm{t}, 0,0, \mathrm{a}(\mathrm{t})\right.$. Thus, $\pi_{1}\left\{w^{4}-\left(\mu_{i}\right)\right\}$ $\approx \pi_{1}\left(\mathrm{~F}_{7} \subset\left\{\mathrm{w}^{4}-\left(\mu_{i}\right)\right\}\right)$ is isomorphic to identity group.

The fundamental group of the deformation retract for the flat Robertson -Walker space $\left\{w^{4}-\left(\mu_{i}\right)\right\}$ onto a geodesic $\mathrm{P}_{2} \subset\left\{w^{4}-\left(\mu_{i}\right)\right\}$ is given by

$\pi_{1}(\eta(\mathrm{m}, \mathrm{c}))=\ln \mathrm{e}^{(1-\mathrm{h})}\{\{\mathrm{t}, \mathrm{a}(\mathrm{t}) \mathrm{r} \sin \theta \cos \phi, \mathrm{a}(\mathrm{t}) \mathrm{r} \sin \theta \sin \phi$, $\left.\mathrm{a}(\mathrm{t}) \mathrm{r} \cos \theta\}-\left(\mu_{\mathrm{i}}\right)\right\}+\ln \mathrm{e}^{\mathrm{n}}\{0,0,0, \mathrm{a}(\mathrm{t}) \mathrm{r}\}$. Thus, $\pi_{1}\left\{w^{4}-\left(\mu_{\mathrm{i}}\right)\right\} \approx$ $\pi_{1}\left(\mathrm{P}_{2} \subset\left\{w^{4}-\left(\mu_{i}\right)\right\}\right)$ is isomorphic to identity group.

The fundamental group of the deformation retract for the flat Robertson -Walker space $\left\{w^{4}-\left(\mu_{i}\right)\right\}$ onto a geodesic $S^{1} \subset\left\{w^{4}-\left(\mu_{i}\right)\right\}$ is given by

$$
\pi_{1}(\eta(\mathrm{m}, \mathrm{c}))=\frac{(-2 \mathrm{c}+2)}{2}\{\{\mathrm{t}, \mathrm{a}(\mathrm{t}) \mathrm{r} \sin \theta \cos \phi, \mathrm{a}(\mathrm{t}) \mathrm{r} \sin \theta
$$

$\left.\sin \phi, a(t) \cos \theta\}-\left(\mu_{\mathrm{i}}\right)\right\}+\mathrm{c}\{0, \mathrm{a}(\mathrm{t}) \mathrm{r} \sin \theta, 0, \mathrm{a}(\mathrm{t}) \mathrm{r} \cos \theta\}$.

Thus, $\pi_{1}\left\{w^{4}-\left(\mu_{i}\right)\right\} \approx \pi_{1}\left(\mathrm{~S}^{1} \subset\left\{w^{4}-\left(\mu_{i}\right)\right\}\right.$ is isomorphic to $\mathrm{Z}$.

Now, we are going to discuss the folding $\mathfrak{I}$ of the flat Robertson-Walker space $\left\{w^{4}-\left(\mu_{i}\right)\right\}$. Let $\mathfrak{I}:\left\{w^{4}-\left(\mu_{i}\right)\right\} \rightarrow$ $\left\{w^{4}-\left(\mu_{i}\right)\right\}$, where

$$
\mathfrak{I}\left(\mathrm{x}_{1}, \mathrm{x}_{2}, \mathrm{x}_{3}, \mathrm{x}_{4}\right)=\left(\mathrm{x}_{1},\left|\mathrm{x}_{2}\right|, \mathrm{x}_{3}, \mathrm{x}_{4}\right)
$$

An isometric folding of flat Robertson -Walker space $\left\{w^{4}-\left(\mu_{i}\right)\right\}$ into itself may be defined by

$\mathfrak{I}:\{(\mathrm{t}, \mathrm{a}(\mathrm{t}) \mathrm{r} \sin \theta \cos \phi, \mathrm{a}(\mathrm{t}) \mathrm{r} \sin \theta \sin \phi$,

$\left.\mathrm{a}(\mathrm{t}) \mathrm{r} \cos \theta)-\left(\mu_{\mathrm{i}}\right)\right\} \rightarrow\{(\mathrm{t},|\mathrm{a}(\mathrm{t}) \mathrm{r} \sin \theta \cos \phi|$,

$\left.\mathrm{a}(\mathrm{t}) \mathrm{r} \sin \theta \sin \phi, \mathrm{a}(\mathrm{t}) \mathrm{r} \cos \theta)-\left(\mu_{i}\right)\right\}$.

The fundamental group of the deformation retract for the folded flat Robertson -Walker space $\pi_{1}\left(\eta \mathfrak{I}\left\{w^{4}-\left(\mu_{i}\right)\right\}\right)$ onto the folded geodesic $\pi_{1}\left(\eta \mathfrak{I}\left(\mathrm{F}_{5}\right)\right)$ is

$\pi_{1}(\eta \mathfrak{I}):\{\{\mathrm{t},|\mathrm{a}(\mathrm{t}) \mathrm{r} \sin \theta \cos \phi|, \mathrm{a}(\mathrm{t}) \mathrm{r} \sin \theta \sin \phi$,

$\left.\mathrm{a}(\mathrm{t}) \mathrm{r} \cos \theta\}-\left(\mu_{i}\right)\right\} \times \mathrm{I} \rightarrow\{\{\mathrm{t},|\mathrm{a}(\mathrm{t}) \mathrm{r} \sin \theta \cos \phi|$,

$\left.\mathrm{a}(\mathrm{t}) \mathrm{r} \sin \theta \sin \phi, \mathrm{a}(\mathrm{t}) \mathrm{r} \cos \theta\}-\left(\mu_{\mathrm{i}}\right)\right\}$

with $\pi_{1}(\eta \mathfrak{I}(\mathrm{m}, \mathrm{c}))=\cos \frac{\pi \mathrm{c}}{2}\{\{\mathrm{t},|\mathrm{a}(\mathrm{t}) \mathrm{r} \sin \theta \cos \phi|, \mathrm{a}(\mathrm{t}) \mathrm{r}$

$\left.\sin \theta \sin \phi, \mathrm{a}(\mathrm{t}) \mathrm{r} \cos \theta\}-\left(\mu_{i}\right)\right\}+\sin \frac{\pi \mathrm{c}}{2}\{\mathrm{t}, 0, \mathrm{a}(\mathrm{t}) \mathrm{r} \sin \theta, \mathrm{a}(\mathrm{t})$

$\mathrm{r} \cos \theta\}$. Thus, $\pi_{1}\left(\eta \mathfrak{I}\left\{w^{4}-\left(\mu_{i}\right)\right\}\right) \approx \pi_{1}\left(\eta \mathfrak{I}\left(\mathrm{F}_{5}\right)\right)$ is isomorphic to identity group.

The fundamental group of the deformation retract for the folded flat Robertson -Walker space $\pi_{1}\left(\eta \mathfrak{I}\left\{w^{4}-\left(\mu_{i}\right)\right\}\right)$ onto the folded geodesic $\pi_{1}\left(\eta \mathfrak{I}\left(\mathrm{F}_{7}\right)\right)$ is $\pi_{1}(\eta \mathfrak{I}(\mathrm{m}, \mathrm{c}))=\frac{1-\mathrm{c}}{1+\mathrm{c}}\{\{\mathrm{t},|\mathrm{a}(\mathrm{t}) \mathrm{r} \sin \theta \cos \phi|, \mathrm{a}(\mathrm{t}) \mathrm{r} \sin \theta$ $\left.\sin \phi, \mathrm{a}(\mathrm{t}) \mathrm{r} \cos \theta\}-\left(\mu_{i}\right)\right\}+\mathrm{c}(2 \mathrm{c}-1)\{\mathrm{t}, 0,0, \mathrm{a}(\mathrm{t}) \mathrm{r}\}$. Thus, $\pi_{1}$ $\left(\eta \mathfrak{I}\left\{w^{4}-\left(\mu_{i}\right)\right\}\right) \approx \pi_{1}\left(\eta \mathfrak{I}\left(\mathrm{F}_{7}\right)\right)$ is isomorphic to identity group.

The fundamental group of the deformation retract for the folded flat Robertson -Walker space $\pi_{1}\left(\eta \mathfrak{I}\left\{w^{4}-\left(\mu_{i}\right)\right\}\right)$ onto the folded geodesic $\pi_{1}\left(\eta \mathfrak{I}\left(S^{1}\right)\right)$ is

$$
\pi_{1}(\eta \mathfrak{I}(\mathrm{m}, \mathrm{c}))=\frac{(-2 \mathrm{c}+2)}{2}\{\{\mathrm{t},|\mathrm{a}(\mathrm{t}) \mathrm{r} \sin \theta \cos \phi|, \mathrm{a}(\mathrm{t}) \mathrm{r} \sin \theta
$$

$\sin \phi, \mathrm{a}(\mathrm{t}) \mathrm{r} \cos \theta\}-(a)\}+\mathrm{c}\{0, \mathrm{a}(\mathrm{t}) \mathrm{r} \sin \theta, 0, \mathrm{a}(\mathrm{t}) \mathrm{r} \cos \theta\}$ Thus, $\pi_{1}\left(\eta \mathfrak{I}\left\{w^{4}-\left(\mu_{i}\right)\right\}\right) \approx \pi_{1}\left(\eta \mathfrak{I}\left(\mathrm{S}^{1}\right)\right)$ is isomorphic to $\mathrm{Z}$.

The fundamental group of the deformation retract for the folded flat Robertson -Walker space $\pi_{1}\left(\eta \mathfrak{I}\left\{w^{4}-\left(\mu_{i}\right)\right\}\right)$ onto the folded geodesic $\pi_{1}\left(\eta \mathfrak{I}\left(\mathrm{P}_{2}\right)\right)$ is

$$
\pi_{1}(\eta \mathfrak{I}(\mathrm{m}, \mathrm{c}))=\ln \mathrm{e}^{(1-\mathrm{h})}\{\{\mathrm{t},|\mathrm{a}(\mathrm{t}) \mathrm{r} \sin \theta \cos \phi|, \mathrm{a}(\mathrm{t}) \mathrm{r} \sin \theta
$$

$\left.\sin \phi, \mathrm{a}(\mathrm{t}) \mathrm{r} \cos \theta\}-\left(\mu_{i}\right)\right\}+\ln \mathrm{e}^{\mathrm{h}}\{0,0,0, \mathrm{a}(\mathrm{t}) \mathrm{r}\}$. Thus, $\pi_{1}$ $\left(\eta \mathfrak{I}\left\{w^{4}-\left(\mu_{i}\right)\right\}\right) \approx \pi_{1}\left(\eta \mathfrak{I}\left(\mathrm{P}_{2}\right)\right)$ is isomorphic to identity group.

Theorem 4. Under the defined folding and any folding homeomorphic to this type of folding, the fundamental group of the deformation retracts of the folded flat Robertson -Walker space i.e. $\pi_{1}\left(\eta \mathfrak{I}^{*}\left\{w^{4}-\left(\mu_{i}\right)\right\}\right)$ into the folded geodesics is different from the fundamental group of the deformation retract of the flat Robertson -Walker space into the geodesics under condition (7).

Proof. Now, let the folding be defined by $\mathfrak{I}^{*}:\left\{w^{4}-\left(\mu_{i}\right)\right\} \rightarrow$ $\left.\left\{w^{4}-\left(\mu_{i}\right)\right\}\right)$, where $\mathfrak{I}^{*}\left(\mathrm{x}_{1}, \mathrm{x}_{2}, \mathrm{x}_{3}, \mathrm{x}_{4}\right)=\left(\mathrm{x}_{1}, \mathrm{x}_{2}, \mathrm{x}_{3},\left|\mathrm{x}_{4}\right|\right)$

The isometric folding of the flat Robertson -Walker space $\mathfrak{I}^{*}\left(\left\{w^{4}-\left(\mu_{i}\right)\right\}\right)$ is

\section{$\overline{\mathrm{R}}=\{\mathrm{t}, \mathrm{a}(\mathrm{t}) \mathrm{r} \sin \theta \cos \phi, \mathrm{a}(\mathrm{t}) \mathrm{r} \sin \theta \sin \phi,|\mathrm{a}(\mathrm{t}) \mathrm{r} \cos \theta|\}$}

The fundamental group of the deformation retract for the folded flat Robertson -Walker space $\pi_{1}\left(\eta \mathfrak{I}^{*}\right.$ $\left.\left\{w^{4}-\left(\mu_{i}\right)\right\}\right)$ onto the folded geodesic $\pi_{1}\left(\eta \mathfrak{J}^{*}\left(\left(\mathrm{~S}^{1}\right)\right)\right)$ is given by

$\pi_{1}\left(\eta \mathfrak{J}^{*}\right):\{\{\mathrm{t}, \mathrm{a}(\mathrm{t}) \mathrm{r} \sin \theta \cos \phi, \mathrm{a}(\mathrm{t}) \mathrm{r} \sin \theta \sin \phi, \mid \mathrm{a}(\mathrm{t})$ $\left.\mathrm{r} \cos \theta \mid\}-\left(\mu_{i}\right)\right\} \times \mathrm{I} \rightarrow\{\{\mathrm{t}, \mathrm{a}(\mathrm{t}) \mathrm{r} \sin \theta \cos \phi, \mathrm{a}(\mathrm{t}) \mathrm{r} \sin \theta \sin \phi, \mid \mathrm{a}(\mathrm{t})$ $\mathrm{r} \cos \theta \mid\}-(\alpha)\}$.

with $\quad \pi_{1}\left(\eta \mathfrak{J}^{*}(\mathrm{~m}, \mathrm{c})\right)=\cos \frac{\pi \mathrm{c}}{2}\{\{\mathrm{t}, \mathrm{a}(\mathrm{t}) \mathrm{r} \sin \theta \cos \phi, \mathrm{a}(\mathrm{t})$ $\left.\mathrm{r} \sin \theta \sin \phi,|\mathrm{a}(\mathrm{t}) \mathrm{r} \cos \theta|\}-\left(\mu_{i}\right)\right\}+\sin \frac{\pi \mathrm{c}}{2}\{0, \mathrm{a}(\mathrm{t}) \mathrm{r} \sin \theta, 0, \mid \mathrm{a}(\mathrm{t})$ $r \cos \theta \mid\}$. Thus, $\pi_{1}\left(\eta \mathfrak{I}^{*}\left\{w^{4}-\left(\mu_{i}\right)\right\}\right) \approx \pi_{1}\left(\eta \mathfrak{I}^{*}\left(S^{1}\right)\right)$ isisomorphic to identity group. 
Theorem 5. The limit folding of the flat Robertson Walker space $\left\{w^{4}-\left(\mu_{i}\right)\right\}$ into itself, under condition (8), is different from the retraction of the flat Robertson -Walker space $\left\{w^{4}-\left(\mu_{i}\right)\right\}$, and the fundamental group of the limit folding is isomorphic to identity group.

Proof. Let $\Pi_{\mathrm{m}}: \mathrm{F} \rightarrow$ Fbe given by: $\Pi_{\mathrm{m}}\left(\mathrm{X}_{1}, \mathrm{X}_{2}, \mathrm{X}_{3}, \mathrm{X}_{4}\right)=\left(\mathrm{X}_{1}\right.$, $\left.\frac{\left|\mathrm{X}_{2}\right|}{\mathrm{m}}, \mathrm{X}_{3}, \frac{\left|\mathrm{X}_{4}\right|}{\mathrm{m}}\right)$

Then, the isometric chain folding of the flat Robertson Walker space F into itself may be defined by

$\Pi_{1}:\{\mathrm{t}, \mathrm{a}(\mathrm{t}) \mathrm{r} \sin \theta \cos \phi, \mathrm{a}(\mathrm{t}) \mathrm{r} \sin \theta \sin \phi$,

$\mathrm{a}(\mathrm{t}) \mathrm{r} \cos \theta\} \rightarrow\{\mathrm{t},|\mathrm{a}(\mathrm{t}) \mathrm{r} \sin \theta \cos \phi|$

$\mathrm{a}(\mathrm{t}) \mathrm{r} \sin \theta \sin \phi,|\mathrm{a}(\mathrm{t}) \mathrm{r} \cos \theta|\}$,

$\Pi_{2}:\{\mathrm{t},|\mathrm{a}(\mathrm{t}) \mathrm{r} \sin \theta \cos \phi|, \mathrm{a}(\mathrm{t}) \mathrm{r} \sin \theta \sin \phi$,

$|\mathrm{a}(\mathrm{t}) \mathrm{r} \cos \theta|\} \rightarrow\left\{\mathrm{t},\left|\frac{\mathrm{a}(\mathrm{t}) \mathrm{r} \sin \theta \cos \phi}{2}\right|\right.$,

$\left.\mathrm{a}(\mathrm{t}) \mathrm{r} \sin \theta \sin \phi,\left|\frac{\mathrm{a}(\mathrm{t}) \mathrm{r} \cos \phi}{2}\right|\right\}, \ldots$,

$\Pi_{\mathrm{m}}:\left\{\mathrm{t},\left|\frac{\mathrm{a}(\mathrm{t}) \mathrm{r} \sin \theta \cos \phi}{\mathrm{m}-1}\right|, \mathrm{a}(\mathrm{t}) \mathrm{r} \sin \theta \sin \phi,\left|\frac{\mathrm{a}(\mathrm{t}) \mathrm{r} \cos \theta}{\mathrm{m}-1}\right|\right\}$

$\rightarrow\left\{\mathrm{t},\left|\frac{\mathrm{a}(\mathrm{t}) \mathrm{r} \sin \theta \cos \phi}{\mathrm{m}}\right|, \mathrm{a}(\mathrm{t}) \mathrm{r} \sin \theta \sin \phi,\left|\frac{\mathrm{a}(\mathrm{t}) \mathrm{r} \cos \theta}{\mathrm{m}}\right|\right\}$

Then we get $\lim _{m \rightarrow \infty} \Pi_{\mathrm{m}}=\{\mathrm{t}, 0, \mathrm{a}(\mathrm{t}) \mathrm{r} \sin \theta \sin \phi, 0\}$, which is hypersurface $\mathrm{F}_{12}$ in flat Robertson -Walker space $\mathrm{F}$. Thus $\pi_{1}\left(\lim _{m \rightarrow \infty} \Pi_{\mathrm{m}}\left\{w^{4}-\left(\mu_{i}\right)\right\}\right) \approx \pi_{1}\left(\mathrm{~F}_{12}\right)$ is isomorphic to identity group.

THEOREM 6. The limit folding of the flat Robertson -Walker space $\left\{w^{4}-\left(\mu_{i}\right)\right\}$ into itself, under condition (9), is equivalent to the zero - dimensional sphere $S^{0}$ in flat Robertson Walker space, and the fundamental group of this type of folding is isomorphic to identity group.

Proof. Let the folding is defined by $\gamma_{\mathrm{n}}:\left\{w^{4}-\left(\mu_{i}\right)\right\} \rightarrow\left\{w^{4}-\right.$ $\left.\left(\mu_{i}\right)\right\}$ such that

$$
\gamma_{\mathrm{n}}\left(\mathrm{x}_{1}, \mathrm{x}_{2}, \mathrm{x}_{3}, \mathrm{x}_{4}\right)=\left(\frac{\left|\mathrm{x}_{1}\right|}{\mathrm{n}}, \frac{\left|\mathrm{x}_{2}\right|}{\mathrm{n}}, \frac{\left|\mathrm{x}_{3}\right|}{\mathrm{n}}, \frac{\left|\mathrm{x}_{4}\right|}{\mathrm{n}}\right)
$$

Then, the isometric chain folding of the flat Robertson Walker space $\left\{w^{4}-\left(\mu_{i}\right)\right\}$ into itself may be defined by $\gamma_{1}:\{\mathrm{t}, \mathrm{a}(\mathrm{t}) \mathrm{r} \sin \theta \cos \phi, \mathrm{a}(\mathrm{t}) \mathrm{r} \sin \theta \sin \phi, \mathrm{a}(\mathrm{t}) \mathrm{r} \cos \theta\} \rightarrow$

$\{|\mathrm{t}|,|\mathrm{a}(\mathrm{t}) \mathrm{r} \sin \theta \cos \phi|,|\mathrm{a}(\mathrm{t}) \mathrm{r} \sin \theta \sin \phi,| \mathrm{a}(\mathrm{t}) \mathrm{r} \cos \theta \mid\}$,

$\gamma_{2}:\{|\mathrm{t}|,|\mathrm{a}(\mathrm{t}) \mathrm{r} \sin \theta \cos \phi|,|\mathrm{a}(\mathrm{t}) \mathrm{r} \sin \theta \sin \phi,| \mathrm{a}(\mathrm{t}) \mathrm{r} \cos \theta \mid\}$,

$\rightarrow\left\{\left|\frac{\mathrm{t}}{2}\right|,\left|\frac{\mathrm{a}(\mathrm{t}) \mathrm{r} \sin \theta \cos \phi}{2}\right|,\left|\frac{\mathrm{a}(\mathrm{t}) \mathrm{r} \sin \theta \sin \phi}{2}\right|,\left|\frac{\mathrm{a}(\mathrm{t}) \mathrm{r} \cos \theta}{2}\right|\right\}, \ldots$,

$\gamma_{\mathrm{n}}:\left\{\left|\frac{\mathrm{t}}{\mathrm{n}-1}\right|,\left|\frac{\mathrm{a}(\mathrm{t}) \mathrm{r} \sin \theta \cos \phi}{\mathrm{n}-1}\right|,\left|\frac{\mathrm{a}(\mathrm{t}) \mathrm{r} \sin \theta \sin \phi}{\mathrm{n}-1}\right|,\left|\frac{\mathrm{a}(\mathrm{t}) \mathrm{r} \cos \theta}{\mathrm{n}-1}\right|\right\}$

$\rightarrow\left\{\left|\frac{\mathrm{t}}{\mathrm{n}}\right|,\left|\frac{\mathrm{a}(\mathrm{t}) \mathrm{r} \sin \theta \cos \phi}{\mathrm{n}}\right|,\left|\frac{\mathrm{a}(\mathrm{t}) \mathrm{r} \sin \theta \sin \phi}{\mathrm{n}}\right|,\left|\frac{\mathrm{a}(\mathrm{t}) \mathrm{r} \cos \theta}{\mathrm{n}}\right|\right\}$

Then we get $\lim _{\mathrm{n} \rightarrow \infty} \gamma_{\mathrm{n}}=\{0,0,0,0\}$, which azero- dimensional hypersurface $S^{0}$ inflatRobertson-Walkerspace $\left\{w^{4}-\left(\mu_{i}\right)\right\}$. Also $\pi_{1}\left(\lim _{\mathrm{n} \rightarrow \infty} \gamma_{\mathrm{n}}\left\{w^{4}-\left(\mu_{i}\right)\right\}\right) \approx \pi_{1}\left(S^{0}\right)$ is isomorphic to identity group.

Theorem 7. Let $\pi_{1}\left(S^{1}\right)$ be a fundamental group of the circle $S^{1}=\{0, \mathrm{a}(t) \mathrm{r} \sin \theta, 0, \mathrm{a}(t) \mathrm{r} \cos \theta\}, \overline{\mathfrak{I}}_{\mathrm{n}}^{1}\left(\pi_{1}\left(S^{1}\right)\right)=\pi_{1}^{\mathrm{n}}\left(S^{1}\right)$ in $\left\{w^{4}-\left(\mu_{i}\right)\right\}$, then there are two types of foldings $\overline{\mathfrak{I}}_{\mathrm{n}}^{\mathrm{i}}\left(\pi_{1}\left(S^{1}\right) \rightarrow \pi_{1}\left(S^{1}\right), \mathrm{i}=1,2\right.$, where n positive integer such that $\overline{\mathfrak{I}}_{\mathrm{n}}^{1}\left(\pi_{1}\left(S^{1}\right)\right)=\pi_{1}^{\mathrm{n}}\left(S^{1}\right)$, and $\overline{\mathfrak{I}}_{\mathrm{n}}^{2}\left(\pi_{1}\left(S^{1}\right)\right)=\pi_{1}\left(S^{1}\right) \bmod \mathrm{n}$.

Proof. First if $\overline{\mathfrak{I}}_{\mathrm{n}}^{1}\left(\pi_{1}\left(S^{1}\right)\right) \rightarrow \pi_{1}\left(S^{1}\right)$ is a folding such that $\overline{\mathfrak{I}}_{\mathrm{n}}^{1}([\alpha])=[\alpha]^{\mathrm{n}}, \mathrm{n}$ positive integer, then $\overline{\mathfrak{I}}_{\mathrm{n}}^{1}\left(\pi_{1}\left(S^{1}\right)\right)=\pi_{1}{ }^{\mathrm{n}}\left(S^{1}\right)$, i.e. $\quad \pi_{1}^{\mathrm{n}}\left(\mathrm{S}^{1}\right)=\left\{[\alpha]^{\mathrm{n}}:[\alpha] \in \pi_{1}\left(\mathrm{~S}^{1}\right)\right\} . \quad$ Also if $\overline{\mathfrak{I}}_{\mathrm{n}}^{2}: \pi_{1}\left(S^{1}\right) \rightarrow$ $\pi_{1}\left(S^{1}\right)$ is a folding such that $\overline{\mathfrak{I}}_{\mathrm{n}}^{2}([\alpha])=([\alpha])$, $\bmod n$ then $\mathfrak{I}_{\mathrm{n}}^{2}\left(\pi_{1}\left(S^{1}\right)\right)=\pi_{1}\left(S^{1}\right) \bmod =\left\{[\alpha]^{k}: k=0,1,2, . ., n-1\right.$, i.e. Where $\alpha$ is a loop.

Theorem 8. There are two types of foldings of the circle $S^{1}=\{0, a(t) r \sin \theta, 0, a(t) r \cos \theta\}$, in $\left\{w^{\uparrow} 4-\left(\mu_{\downarrow} i\right)\right\}$, $\mathfrak{I}_{\mathrm{i}}: S_{1}^{1} \rightarrow S_{2}^{1}, \mathrm{i}=1,2$ without singularities which induce foldings $\overline{\mathfrak{I}}_{\mathrm{i}}: \pi_{1}\left(S_{1}^{1}\right) \rightarrow \pi_{1}\left(S_{2}^{1}\right)$ such that $\overline{\mathfrak{I}}_{\mathrm{i}}\left(\pi_{1}\left(S_{1}^{1}\right)\right)=\pi_{1}\left(\overline{\mathfrak{I}}_{\mathrm{i}}\left(S_{1}^{1}\right)\right)$.

Proof. Let $\mathfrak{I}_{1}: S_{1}^{1} \rightarrow S_{2}^{1}$ be a folding such that $\mathfrak{I}_{1}\left(\mathrm{e}^{\mathrm{i} \theta}\right)=\mathrm{re} \mathrm{e}^{\mathrm{i} \theta}$, $r \in(0, \infty), 0 \leq \theta<2 \pi$ then there is an induced folding $\quad \overline{\mathfrak{I}}_{1}: \pi_{1}\left(\mathrm{~S}_{1}^{1}\right) \rightarrow \pi_{1}\left(\mathrm{~S}_{2}^{1}\right)$ such that $\overline{\mathfrak{I}}_{1}([\alpha])=[\mathrm{r} \alpha]$, where $\alpha=\mathrm{e}^{\mathrm{i}(2 \mathrm{~m} \pi \theta)}, \mathrm{m} \in \mathrm{Z}$. And so $\overline{\mathfrak{I}}_{1}\left(\pi_{1}\left(\mathrm{~S}_{1}^{1}\right)\right)=\pi_{1}\left(\mathfrak{I}_{1}\left(\mathrm{~S}_{2}^{1}\right)\right)$. Also, let $\mathfrak{I}_{2}: \mathrm{S}_{1}^{1} \rightarrow \mathrm{S}_{2}^{1}$ be a folding such that $\mathfrak{I}_{2}\left(\mathrm{e}^{\mathrm{i} \theta}\right)=\mathrm{e}^{\mathrm{i} \phi}, 0 \leq \theta<2 \pi$, , $0 \leq \varphi-\theta<2 \pi$, then there is an induced folding $\overline{\mathfrak{I}}_{2} \pi_{1}\left(\mathrm{~S}_{1}^{1}\right) \rightarrow$ $\pi_{1}\left(\mathrm{~S}_{2}^{1}\right)$ such that $\overline{\mathfrak{I}}_{2}([\alpha])=[\beta]$, where $\alpha=\mathrm{e}^{\mathrm{i}(2 \mathrm{~m} \pi \theta)}, \beta=\mathrm{e}^{\mathrm{i}(2 \mathrm{~m} \pi \varphi)}$, $\mathrm{m} \in \mathrm{Z}$, and so $\overline{\mathfrak{I}}_{2}\left(\pi_{1}\left(\mathrm{~S}_{1}^{1}\right)\right)=\pi_{1}\left(\overline{\mathfrak{I}}_{2}\left(\mathrm{~S}_{2}^{1}\right)\right)$.

Theorem 9. The fundamental group of any folding of $S^{1}=\{0, a(t) r \sin \theta, 0, a(t) r \cos \theta\}$, in $\left\{w^{\uparrow} 4-\left(\mu_{\downarrow} i\right)\right\}$, is either isomorphic to $Z$ or identity group. 
Proof. First, for folding with singularity of $S^{1}=\{0, a(t)$ $r \sin \theta, 0, a(t) r \cos \theta\}$, as in Figure (1) (a), then clearly $\pi_{1}\left(\mathfrak{I}\left(S^{1}\right)\right)=0$. Also, folding without singularity of $S^{1}$, then $\mathfrak{I}\left(\mathrm{S}^{1}\right)$ is a manifold homeomorphic to $S^{1}$ as in Figure (1) (b), and so $\pi_{1}\left(\mathrm{~S}^{1}\right) \approx \mathrm{Z}$.

Theorem 10. Under the condition $\mathrm{X}_{1}=0$, in $\left\{w^{\mathrm{n}+1}-\right.$ $\left.\left(\mu_{\downarrow} i\right)\right\}$. The fundamental group of any folding of $S^{\mathrm{n}}$ such that $\operatorname{dim} \mathfrak{I}\left(S^{\mathrm{n}}\right)=\operatorname{dim}\left(S^{\mathrm{n}}\right), \mathrm{n} \geq 2$, is the identity group.

Proof. First, consider the spherical coordinate by a(t) $r$ and $\theta_{1}, \theta_{2} \ldots, \theta_{\mathrm{n}}$, one has

$x_{1}=a(t) r \pi_{k=1}^{n-1} \sin \theta_{k}$,

$x_{i}=a(t) r \cos \theta_{i-1} \pi_{k=i}^{n-1} \sin \theta_{k}, 1<i<n+1$

$x_{n+1}=a(t) r \cos \theta_{n}$.

for folding with singularity of $S^{n}$ to a subset of $S^{n}$ with $\operatorname{dim} \mathfrak{I}\left(S^{n}\right)=\operatorname{dim}\left(S^{n}\right)$ as in Figure (2) (a). Then all loops of $\mathfrak{I}\left(S^{\mathrm{n}}\right)$ are homotopic to the identity loop, and so the fundamental group of it is folding is the identity group. Also, folding $S^{\mathrm{n}}$ without singularity to a manifold homeomorphic to $\mathrm{S}^{\mathrm{n}}$ as in Figure (2) (b) and so the fundamental group is the identity group.

Theorem 11. Under the condition $X_{1}=0$. Let $D_{n}$ be the disjoint union of $\mathrm{n}$ discs on $\mathrm{S}^{2}=(0, a(t) r \sin \theta \cos \phi, a(t) r$ $\sin \theta \sin \phi, a(t) r \cos \theta)$ in flat Robertson-Walker space with radius $(\mathrm{a}(\mathrm{t}) \mathrm{r})$. Then the fundamental group of any folding of $S^{2}-D_{n}$ into itself is a free group of rank $\leq n-1$.

Proof. Let $\mathfrak{I}:\left(S^{2}-D_{n}\right) \rightarrow\left(S^{2}-D_{n}\right)$ be any folding of $S^{2}-$ $D_{n}$ into itself then $\mathfrak{I}\left(S^{2}-D_{n}\right)$ is a space homeomorphic to $S^{2}-$ $\mathrm{D}_{\mathrm{k}}, \mathrm{k} \leq \mathrm{n}$, thus $\pi_{1}\left(\mathfrak{I}\left(\mathrm{S}^{2}-\mathrm{D}_{\mathrm{n}}\right)\right) \approx \pi_{1}\left(\mathfrak{I}\left(\mathrm{S}^{2}-\mathrm{D}_{\mathrm{k}}\right)\right)$. But $(\mathrm{k}-1)$ leaved rose is a deformation retract of $S^{2}-D_{k}$ as in Figure (3) for $\mathrm{n}=2$, so we have $\pi_{1}\left(S^{2}-D_{k}\right) \approx \underbrace{\pi_{1}\left(S^{1} \vee S^{1} \vee \ldots \vee S^{1}\right)}_{k-1 \text { terms }}$,

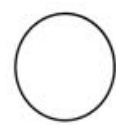

Folding with singularity
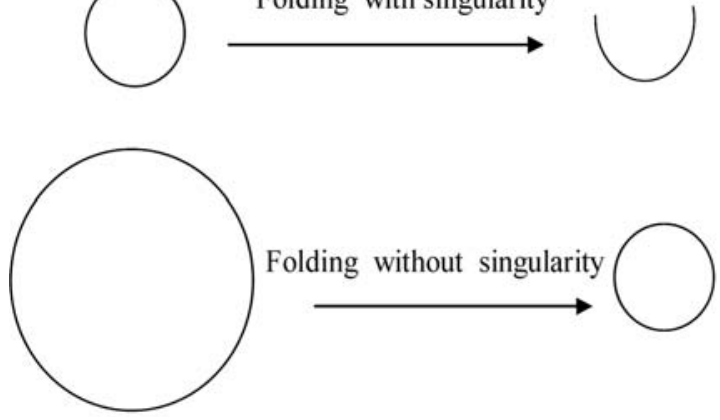

( a)

(b)

Figure 1. Represented types of folding with singularity of the circle and folding without singularity. where $\underbrace{S^{1} \vee S^{1} \vee \ldots S^{1}}_{k-1}$ denote the wedge sum of $(k-1)$ circles and so $\pi_{1}\left(\mathrm{~S}^{2}-\mathrm{D}_{\mathrm{k}}\right) \approx \underbrace{\pi_{1}\left(\mathrm{~S}^{1}\right) * \pi_{1}\left(\mathrm{~S}^{1}\right) \cdots \pi_{1}\left(\mathrm{~S}^{1}\right)}_{k-1 \text { terms }} \approx \underbrace{Z * Z * \cdots * Z}_{k-1 \text { terms }}$. Thus $\pi_{1}\left(S^{2}-D_{k}\right)$ is a free group of rank $k-1$. Since $\pi_{1}$ $\left(\mathfrak{I}\left(\mathrm{S}^{2}-\mathrm{D}_{\mathrm{n}}\right)\right) \approx \pi_{1} \mathfrak{I}\left(\mathrm{S}^{2}-\mathrm{D}_{\mathrm{k}}\right)$ it follows that $\pi_{1}\left(\mathfrak{I}\left(\mathrm{S}^{2}-\mathrm{D}_{\mathrm{n}}\right)\right)$ is a free group of rank $k-1$. Therefore $\pi_{1}\left(\mathfrak{I}\left(S^{2}-D_{n}\right)\right)$ is a free group of rank $\leq \mathrm{n}-1$.

Theorem 12. Let $\mathrm{X} \subset\left\{\mathrm{W}^{2}-\left(\mu_{i}\right)\right\}$ be the union of the circles $\mathrm{C}_{\mathrm{n}}$ of radii $\frac{1}{\mathrm{a}(\mathrm{t}) \mathrm{r}}$ and centered at $\left(\frac{1}{\mathrm{a}(\mathrm{t}) \mathrm{r}}, 0\right), n=1,2, \ldots$, then there are foldings $\mathfrak{I}_{n}: X \rightarrow X$ and retractions $\mathrm{R}_{\mathrm{n}}: \mathrm{X} \rightarrow \mathrm{C}_{\mathrm{n}}$ such that $\pi_{1}\left(\mathfrak{I}_{n}(X)\right)=\pi_{1}\left(R_{n}(X)\right)$ and $\pi_{1}\left(\mathfrak{I}_{n}(X)\right)$ is either identity group or isomorphic to $Z$.

Proof. Let $\mathfrak{I}_{\mathrm{n}}: X \rightarrow X$ be a folding such that $\mathfrak{I}_{\mathrm{n}}\left(C_{\mathrm{m}}\right)=C_{\mathrm{n}}, \forall \mathrm{m}=1,2$,

where $C_{m}=\left\{a_{m}(t) r_{m} \sin \theta, a_{m}(t) r_{m} \cos \theta, \forall m=1,2,\right\}$, then, $3_{n}(X)=C_{n}$ as in Figure (4). Also, Consider the retractions $R_{n}: X \rightarrow C_{n}$, which collapsing all $C_{i}$ except $C_{n}$ to the origin and so $R_{n}(X)=C_{n}$ then $\pi_{1}\left(\mathfrak{I}_{n}(X)\right)=\pi_{1}\left(R_{n}(X)\right)$. Now, if $\mathrm{n} \rightarrow \infty$ then $\mathrm{C}_{\mathrm{n}}$ is a point and so $\pi_{1}\left(\mathfrak{I}_{\mathrm{n}}(\mathrm{X})\right)=0$. Otherwise if $n \nrightarrow \infty$ then $C_{n}$ is a circle and clearly $\pi_{1}\left(\mathfrak{I}_{n}(X)\right) \approx Z$. Therefore, $\pi_{1}\left(\mathfrak{I}_{\mathrm{n}}(\mathrm{X})\right)$ is either identity group or isomorphic to $\mathrm{Z}$.
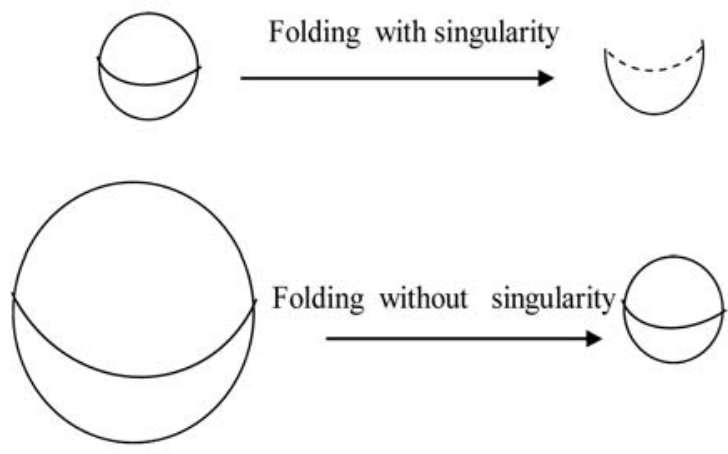

Figure 2. Represented types of folding with singularity and folding without singularity.

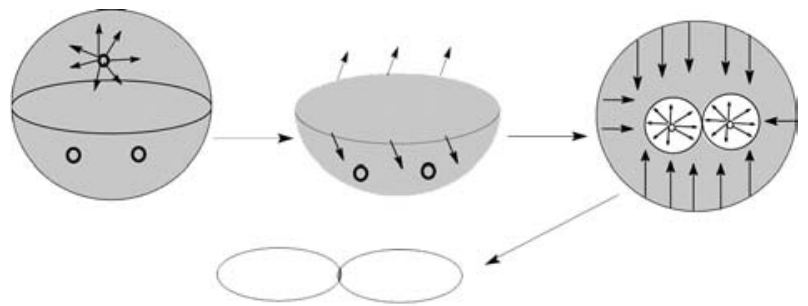

Figure 3. Represented types of the disjoint union of discs on the sphere. 


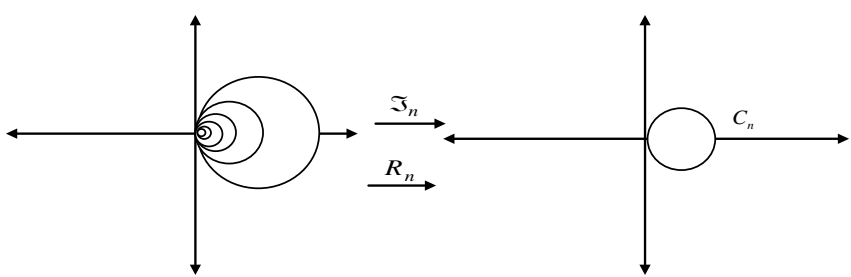

Figure 4. Represented types of the the union of the circles $\mathrm{C}_{\mathrm{n}}$ of radii $\frac{1}{\mathrm{a}(\mathrm{t}) \mathrm{r}}$ and centered at $\left(\frac{1}{\mathrm{a}(\mathrm{t}) \mathrm{r}}, 0\right)$.

Theorem 13. Let $\mathrm{X} \subset \mathrm{W}^{3}$ be the union of the spheres $\mathrm{S}_{\mathrm{n}}^{2}-\left\{a_{1}, a_{2}\right\}=((0, a(t) r \sin \theta \cos \phi, a(t) r \sin \theta \sin \phi, a(t) r \cos \theta)$ $\left.-\left\{a_{1}, a_{2}\right\}\right) a_{1}, a_{2} \notin \bigcap_{n=1}^{\infty} S_{\mathrm{n}}^{2}$ of radii $\frac{1}{a(t) r}$ and centered at $\left(\frac{1}{a(t) r}, 0,0\right), n=1,2, .$, then there are foldings $\mathfrak{I}_{n}: \mathrm{X} \rightarrow \mathrm{X}$ and retractions $\mathrm{R}_{\mathrm{n}}: \mathrm{X} \rightarrow \mathrm{S}_{\mathrm{n}}^{2}-\left\{a_{1}, a_{2}\right\}$ such that $\pi_{1}\left(\mathfrak{I}_{n}(X)\right)=$ $\pi_{1}\left(R_{n}(X)\right)$ and $\pi_{1}\left(\mathfrak{I}_{n}(X)\right)$ is either identity group or isomorphic to Z.

\section{Conclusion}

In this paper we achieved the approval of the important of the fundamental groups in the submanifolds of flat Robertson-Walker space by using some geometrical transformations. The relations between folding, retraction, deformation retract, limits of foldings and limits of retractions of the fundamental groups in the submanifolds of flat Robertson-Walker space are discussed. The connection between limits of the foldings and the fundamental groups are obtained. New types of minimal retractions on the fundamental groups are deduced.

\section{Acknowledgments}

The author is deeply indebted to the team work at the deanship of the scientific research, Taibah University for their valuable help and critical guidance and for facilitating many administrative procedures. This research work was financed supported by Grant no. 3066/1434 from the deanship of the scientific research at Taibah University, Al-Madinah Al-Munawwarah, Saudi Arabia.

\section{References}

1. El-Ahmady A E (2007). The variation of the density on chaotic spheres in chaotic space-like Minkowski space time, Chaos, Solitons \& Fractals, vol 31(5), 1272-1278.
2. El-Ahmady A E (2007). Folding of fuzzy hypertori and their retractions, Proc. Math. Phys. Soc. Egypt, vol 85(1), $1-10$.

3. El-Ahmady A E (2006). Limits of fuzzy retractions of fuzzy hyperspheres and their foldings, Tamkang Journal of Mathematics, vol 37(1), 47-55.

4. El-Ahmady A E (2004). Fuzzy folding of fuzzy horocycle, Rendiconti del Circolo Matematico di Palermo Serie II, Tomo L III, vol 53(3), 443-450.

5. El-Ahmady A E (2004). Fuzzy Lobachevskian space and its folding, Journal of Fuzzy Mathematics, vol 12(2), 609-614.

6. El-Ahmady A E (1994). The deformation retract and topological folding of Buchdahi space, Periodica Mathematica Hungarica, vol 28(1), 19-30.

7. El-Ahmady A E (2011). The geodesic deformation retract of Klein bottle and its folding, International Journal of Nonlinear Science, vol 12(3), 323-330.

8. El-Ahmady A E (2013). Folding and fundamental groups of Buchdahi space, Indian Journal of Science and Technology, vol 6(1), 3940-3945.

9. El-Ahmady A E (In press). Folding of some types of Einstein spaces, The International Journal of Nonlinear Science.

10. El-Ahmady A E. On elastic Klein bottle and fundamental groups, Applied Mathematics, (Accepted).

11. El-Ahmady A E (2011). Retraction of chaotic black hole, The Journal of Fuzzy Mathematics, vol 19(4), 833-838.

12. El-Ahmady A E (2013). On the fundamental group and folding of Klein bottle, International Journal of Applied Mathematics and Statistics, vol 37( 6), 56-64.

13. El-Ahmady A E. Fuzzy elastic Klein bottle and its retractions International Journal of Applied Mathematics and Statistics, (Accepted).

14. El-Ahmady A E, and Al-Luhaybi A S (2013). A Calculation of geodesics in flat Robertson-Walker space and its folding, International Journal of Applied Mathematics and Statistics, vol 33(3), 83-91.

15. El-Ahmady A E, and A S Al-Luhaybi (2013). Fuzzy retractions of fuzzy open flat Robertson-Walker space, Advances in Fuzzy Systems, vol 2013, 1-7.

16. El-Ahmady A E, and Al-Luhaybi A S (2012). Ageometrical characterization of spatially curved Robertson-Walker space and its retractions, Applied Mathematics, vol 12(3), 1153-1160.

17. El-Ahmady A E, and Al-Luhaybi A S (2012). Retractions of spatially curved Robertson-Walker space, The Journal of American Sciences, vol 8(5), 548-553.

18. El-Ahmady A E, and Al-Luhaybi A S. On fuzzy retracts of fuzzy closed flat Roberstion-Walker spaces, Advances in fuzzy sets and systems,(Accepted). 
19. El-Ahmady A E, and Al-Luhaybi A S. Retractions of fuzzy flat Robertson-Walker space, International Journal of Applied Mathematics and Statistics,(Accepted).

20. Naber G L (2011). Topology, Geometry and Gauge fields, Foundations, New York, Berlin.

21. Hartle J B (2003). Gravity, An introduction to Einstein's general relativity, Addison-Wesley, New York.

22. El-Ahmady A E (2012). Folding and unfolding of chaotic spheres in chaotic space-like Minkowski spacetime, The Scientific Journal of Applied Research, vol 1(2), 34-43.

23. El-Ahmady A E (2012). Retraction of null helix in Minkowski 3-space, The Scientific Journal of Applied Research, vol 1(2), 28-33.
24. El-Ahmady A E, and Al-Hazmi N (2013). Foldings and deformation retractions of hypercylinder, Indian Journal of Science and Technology, vol 6(2), 4084-4093.

25. Abu-Saleem M (2007). Some geometric transformations on manifolds and their algebraic structures, Ph. D. Thesis, Tanta University, Egypt.

26. Reid M, and Szendroi B (2005). Topology and geometry, Cambridge, New York.

27. Arkowitz M (2011). Introduction to homotopy theory, Springer-Verlage, New York.

28. Shick P L (2007). Topology: Point-Set and geometry, New York, Wiley.

29. Strom, J (2011). Modern classical homotopy theory, American Mathematical Society. 